\title{
LORENTZIAN GEOMETRY IN THE LARGE
}

\author{
JOHN K. BEEM \\ Mathematics Department, University of Missouri-Columbia \\ Columbia, MO 65211, U.S.A. \\ E-mail: mathjkb@showme.missouri.edu
}

\begin{abstract}
Lorentzian geometry in the large has certain similarities and certain fundamental differences from Riemannian geometry in the large. The Morse index theory for timelike geodesics is quite similar to the corresponding theory for Riemannian manifolds. However, results on completeness for Lorentzian manifolds are quite different from the corresponding results for positive definite manifolds. A generalization of global hyperbolicity known as pseudoconvexity is described. It has important implications for geodesic structures.
\end{abstract}

1. Introduction. The Hopf-Rinow Theorem [13], [14] is a key result for Riemannian (i.e., positive definite) manifolds. It yields the equivalence for these manifolds of Cauchy completeness (of the induced distance function), geodesic completeness and a third type of completeness known as finite compactness (i.e., bounded sets have compact closure). It also guarantees the existence of at least one geodesic joining any two distinct points of a complete Riemannian manifold. This last property is known as geodesic connectedness [21]. Furthermore, the equivalence of finite compactness and Cauchy completeness easily yields the completeness of all compact Riemannian manifolds. Thus, compact Riemannian manifolds are both complete and geodesically connected. The situation for Lorentzian manifolds is quite different. If one has a space-time (i.e., a time-oriented Lorentzian manifold) then one has a Lorentzian distance function. However, this function does not do as much as the corresponding distance function for positive definite manifolds. In the first place, the Lorentzian distance function is only positive for points connected by timelike curves. In this case the distance is given as the supremum of lengths of causal curves from the first point to the second. Notice that this Lorentzian distance is not necessarily finite valued and is not a true distance in the topological sense. Also, one does not have various forms of completeness equivalent to geodesic completeness. Compact space-times are generally considered to be of marginal physical importance since each compact space-time admits a closed timelike curve. Nevertheless, they are still

1991 Mathematics Subject Classification: Primary 53C50; Secondary 83C99.

The paper is in final form and no version of it will be published elsewhere. 
of mathematical interest. However, examples show that they need not be geodesically complete [2] and need not be geodesically connected [6].

In spite of a number of differences, there are still many ways in which the global theory for Lorentzian manifolds is quite parallel to the corresponding theory for Riemannian manifolds. One example of similar results is found in the splitting theorems, compare [9], [10], [20]. However, it should be mentioned that for results such as the splitting theorems, the proofs in the Lorentzian case can involve a number of new difficulties. In Section 2 we consider Jacobi fields and index theory along geodesics. Some of the ways in which global theory of Lorentzian [2] and Riemannian [11] manifolds are similar are given in this section. In particular, we give the Morse index theory for timelike geodesics which says that the index form may be used to count conjugate points along a geodesic. Also, we give a timelike diameter result which parallels the usual Diameter Theorem familiar in the study of positive definite manifolds.

In Section 3 we consider an internal type of completeness property known as pseudoconvexity. This property has applications in terms of obtaining sufficient conditions for the $C^{1}$-fine stability of geodesic completeness and in terms of a Lorentzian version of the Hadamard-Cartan Theorem. We also use pseudoconvexity in Section 4 where the geodesics of a given $(M, g)$ are made into a space $G(M)$ by identifying each geodesic as a single element of $G(M)$. If the geodesics of $(M, g)$ satisfy a nonreturning property, then the geodesic system is pseudoconvex iff $G(M)$ is a $2 n-2$ dimensional manifold.

It should also be mentioned that one has a rich structure of problems for Lorentzian manifolds that have no direct parallel in the positive definite case. For example, problems dealing with black holes [12], [16] and cosmic censorship [8], [17] do not arise in the positive definite case.

2. Jacobi fields and index forms. Let $(M, g)$ be a space-time with metric signature of the form $(-,+,+,+)$, with induced Levi-Civita connection $\nabla$, and with curvature tensor

$$
R(X, Y) Z=\nabla_{X} \nabla_{Y} Z-\nabla_{Y} \nabla_{X} Z-\nabla_{[X, Y]} Z .
$$

Given a geodesic $\gamma:(a, b) \rightarrow M$, let $J$ be vector field along $\gamma$. The vector field $J$ is called a Jacobi field [2] if it satisfies the following equation

$$
J^{\prime \prime}+R\left(J, \gamma^{\prime}\right) \gamma^{\prime}=0
$$

Geometrically the vector field $J$ measures the separation of two nearby geodesics. If we further assume that the geodesic $\gamma$ is a unit speed timelike geodesic, then the second derivative $J^{\prime \prime}$ represents the relative or tidal acceleration of two nearby freely falling observers. Using the above Jacobi equation, one obtains

$$
J^{\prime \prime}=-R\left(J, \gamma^{\prime}\right) \gamma^{\prime}
$$

hence

$$
g\left(J^{\prime \prime}, J\right)=-g\left(R\left(J, \gamma^{\prime}\right) \gamma^{\prime}, J\right)
$$

To relate $J^{\prime \prime}$ to curvature, we assume for convenience that $\gamma$ is a unit speed geodesic and that $J$ is orthogonal to the tangent vector $\gamma^{\prime}$. Then, using $g\left(\gamma^{\prime}, \gamma^{\prime}\right)=-1$ and $g\left(J, \gamma^{\prime}\right)=0$ 
one finds the sectional curvature [2], [7] of the plane containing $J$ and $\gamma^{\prime}$ is given by

$$
K\left(J, \gamma^{\prime}\right)=g\left(R\left(J, \gamma^{\prime}\right) \gamma^{\prime}, J\right) /\left[g(J, J) g\left(\gamma^{\prime}, \gamma^{\prime}\right)-g\left(J, \gamma^{\prime}\right)^{2}\right]=-g\left(R\left(J, \gamma^{\prime}\right) \gamma^{\prime}, J\right) / g(J, J)
$$

Using $|J|=g(J, J)^{1 / 2}$, one obtains

$$
g\left(J^{\prime \prime}, J /|J|\right)=K\left(J, \gamma^{\prime}\right)|J| .
$$

The left hand side of the above equation represents the radial component of the tidal acceleration. Consequently, one finds that radial tidal acceleration is proportional to the product of the distance apart (i.e., $|J|$ ) and to the sectional curvature of the plane determined by the tangent vector $\gamma^{\prime}$ and the Jacobi vector $J$. Notice that, with our sign convention, negative sectional curvature for timelike planes has a converging effect on timelike geodesics and positive sectional curvature for timelike planes has a diverging effect. In particular, the above equation and the fact that timelike geodesics may be used to approximate null geodesics arbitrarily closely yield Proposition 2.1 below. Recall that two points are said to be conjugate along a geodesic $\gamma$ if there is a nontrivial Jacobi field which vanishes at both points.

Proposition 2.1. Let $(M, g)$ be a Lorentzian manifold with everywhere nonnegative sectional curvatures on timelike planes, then no causal geodesic has conjugate points.

Since the sectional curvature function generically becomes unbounded near null sections [3], the above equation relating tidal accelerations to sectional curvature implies that, generically, the tidal accelerations experienced by an extended body moving close to light speed must be very large, compare [7], [18], [19]. In fact, they must become unbounded for such extended bodies as they approach light speed unless the null directions their tangent vectors are approaching satisfy certain very special conditions, compare [3], [4], [7].

If $\gamma:[a, b] \rightarrow M$ is a timelike geodesic segment, let $V_{0}^{\perp}$ be the space of all vector fields along $\gamma$ which are always perpendicular to $\gamma^{\prime}$ and which vanish at both endpoints of $\gamma$. Let $X$ and $Y$ belong to $V_{0}^{\perp}$. The timelike index form is defined to be

$$
I(X, Y)=-\int\left[g\left(X^{\prime}, Y^{\prime}\right)-g\left(R\left(X, \gamma^{\prime}\right) \gamma^{\prime}, Y\right)\right] d t .
$$

The index $\operatorname{Ind}(\gamma)$ is defined by

$$
\operatorname{Ind}(\gamma)=\operatorname{lub}\left\{\operatorname{dim}(A) \mid A \text { a linear subspace of } V_{0}^{\perp}, I \text { positive definite on } A\right\}
$$

and $\operatorname{Ind}_{0}(\gamma)$ is defined by

$$
\operatorname{Ind}_{0}(\gamma)=\operatorname{lub}\left\{\operatorname{dim}(A) \mid A \text { a linear subspace of } V_{0}^{\perp}, I \text { positive semi-definite on } A\right\} \text {. }
$$

Let $J_{t}(\gamma)$ is the space of Jacobi fields that vanish at $\gamma(a)$ and $\gamma(t)$. Using the above notation, the Timelike Morse Index Theorem [2] is then given by

THeOrem 2.2. If $(M, g)$ is a space-time and $\gamma:[a, b] \rightarrow M$ is a timelike geodesic, then $\operatorname{Ind}(\gamma)$ counts the conjugate points with multiplicity for $a<t<b$ and Ind $d_{0}(\gamma)$ counts the conjugate points with multiplicity for $a<t \leq b$. More precisely, $\operatorname{Ind}(\gamma)=\sum \operatorname{dim}\left(J_{t}(\gamma)\right)$ where the sum is for $a<t<b$ and $\operatorname{Ind}_{0}(\gamma)=\sum \operatorname{dim}\left(J_{t}(\gamma)\right)$ where the sum is for $a<t \leq b$. 
Recall that for timelike vectors, negative timelike sectional curvature corresponds to positive Ricci curvature. In some sense, the Ricci curvature on a timelike vector $V$ is -3 times the average sectional curvature of timelike planes containing $V$. More precisely, for a timelike vector $V$ the Ricci curvature $\operatorname{Ric}(V, V)=R_{i j} V^{i} V^{j}$ is the negative of the sum of the sectional curvatures of any three timelike planes containing $V$ which have mutually orthogonal normals. The next conjugate point result follows for Lorentzian manifolds [2] from the definition of the index form and the Timelike Morse Index Theorem.

THEOREM 2.3. If $(M, g)$ is a space-time and satisfies either (1) all timelike sectional curvatures satisfy $K_{\text {timelike }} \leq-k<0$ or (2) Ric $(V, V) \geq 3 k>0$ for all timelike unit vectors $V$, then each timelike geodesic $\gamma:[0, b) \rightarrow M$ with $b>\pi / \sqrt{k}$ has a conjugate point to $\gamma(0)$.

The causal future $J^{+}(p)$ of a point $p$ is the set of points that can be reached by a future directed causal curve. The causal past $J^{-}(p)$ is defined dually. A space-time is said to be strongly causal if each point has arbitrarily small neighborhoods such that any causal curve leaving one of these neighborhoods fails to return. Strong causality is usually considered to be a physically realistic assumption. Clearly, its failure raises the possibility of serious physical paradoxes. A globally hyperbolic space-time is a strongly causal space-time which has $J^{+}(p) \cap J^{-}(q)$ compact for all $p, q \in M$. Global hyperbolicity is a type of completeness and a fundamental result in global Lorentzian geometry is that any two timelike related points in a globally hyperbolic space-time may be joined by a timelike geodesic which is of maximal length among all causal curves joining the points [22]. Since the distance between two timelike related points is the supremum of lengths of causal curves joining the points, it follows that the distance between any two timelike related points in a globally hyperbolic space-time is the length of such a maximal timelike geodesic. The timelike diameter, $\operatorname{diam}(M)$, of a Lorentzian manifold $(M, g)$ is defined to be the supremum of distances $d(p, q)$ between points of $M$. A corollary of the last theorem is the Lorentzian version of the Diameter Theorem.

COROLlary 2.4. If $(M, g)$ is a globally hyperbolic space-time and satisfies either (1) all timelike sectional curvatures satisfy $K_{\text {timelike }} \leq-k<0$ or (2) $\operatorname{Ric}(V, V) \geq 3 k>0$ for all timelike unit vectors $V$, then $\operatorname{diam}(M) \leq \pi / \sqrt{k}$.

Proof. Assume there are two points $p$ and $q$ with $d(p, q)=b>\pi / \sqrt{k}$. Global hyperbolicity implies there is a geodesic segment $\gamma:[0, b] \rightarrow M$ which is maximal and goes from $p$ to $q$. However, Theorem 2.3 implies such a geodesic must have a pair of conjugate points and thus fail to be maximal, in contradiction.

3. Pseudoconvexity and disprisonment. Let $\gamma:(a, b) \rightarrow M$ be a geodesic. If $a<t_{0}<b$, then the image corresponding to the forward end (i.e., $\left.\gamma\left[t_{0}, b\right)\right)$ may or may not be imprisoned in a compact set and this also holds for the other end (i.e., $\left.\gamma\left(a, t_{0}\right]\right)$. Thus, a geodesic may have no compactly imprisoned ends, one compactly imprisoned end, or both ends compactly imprisoned. The space-time $(M, g)$ is said to be causally disprisoning [6] if each end of each causal geodesic fails to be imprisoned in any compact set. Furthermore, $(M, g)$ is said to be causally pseudoconvex if for each compact set $K$ 
there is another compact set $H$ such that any causal geodesic segment of $(M, g)$ with both endpoints in $K$ has an image entirely in $H$. This is an "internal" type of completeness. For example, it fails for Minkowski space-time less a single point. In this case, one may take $K$ to be three dimensional surface of a Euclidean ball about the deleted point and easily verify that no compact set $H$ contains all causal geodesic segments joining points of $K$. The next lemma shows that causal pseudoconvexity is a generalization of global hyperbolicity.

LEMMA 3.1. If $(M, g)$ is a globally hyperbolic space-time then $(M, g)$ is both causally disprisoning and causally pseudoconvex.

P r o of. Strong causality yields the nonimprisonment of future and/or past endless causal curves in compact sets [12]. Thus, causal disprisonment must hold. Let $K$ be a given compact set and cover $K$ with open sets of the form $I^{+}(p) \cap I^{-}(q)$ where $I^{+}$and $I^{-}$ denote chronological future and past. Since $K$ is compact there must be a finite subcover of the form $I^{+}\left(p_{i}\right) \cap I^{-}\left(q_{i}\right)$ for $1 \leq i \leq m$. Let $H$ be the finite union of all sets of the form $J^{+}\left(p_{i}\right) \cap J^{-}\left(q_{k}\right)$ where $1 \leq i, k \leq m$. Since $H$ is a finite union of compact sets, the set $H$ is compact. Also, it is an easy matter to verify that all causal curves starting and ending in $K$ must remain in $H$ which implies that all causal geodesic segments with endpoints in $K$ must lie in $H$, as desired.

Let $\operatorname{Lor}(M)$ denote the space of all Lorentzian metrics on $M$. The fine $C^{r}$-topologies are defined on $\operatorname{Lor}(M)$ using a fixed locally finite set of charts $\left\{\left(U_{\alpha}, \phi_{\alpha}\right)\right\}$ such that each of these charts has compact closure in a larger chart. For the covering $\left\{\left(U_{\alpha}, \phi_{\alpha}\right)\right\}$ let $\delta: M \rightarrow(0, \infty)$ be continuous and let

$$
|g-h|_{r}<\delta
$$

mean that all components and mixed partials up to order $r$ for $g$ and $h$ are $\delta$ close in the special covering $\left\{\left(U_{\alpha}, \phi_{\alpha}\right)\right\}$. A basis $\{W(h, \delta)\}$ for the fine $C^{r}$-topology on $\operatorname{Lor}(M)$ is obtained by taking Lorentzian metrics $h$ and continuous functions $\delta: M \rightarrow(0, \infty)$ and setting

$$
W(h, \delta)=\left\{g|| g-\left.h\right|_{r}<\delta\right\} .
$$

A property is said to be $C^{r}$-stable if the set of metrics for which it holds is an open set in the $C^{r}$-topology on $\operatorname{Lor}(M)$. Notice that $C^{r}$-stable implies $C^{s}$-stable for all $r<s$ since each open set in the $C^{r}$-topology is also open in the $C^{s}$-topology. Of course, one may also define these topologies and corresponding notions of stability on other collections of tensors such as the collection $\operatorname{Riem}(M)$ of all Riemannian metrics on $M$. Interestingly, both geodesic completeness and geodesic incompleteness are $C^{0}$-stable for $\operatorname{Riem}(M)$. This follows easily since if $g$ is a positive definite metric on $M$ which is complete (respectively, incomplete), then the set $W$ in $\operatorname{Riem}(M)$ consisting of all metrics $h$ satisfying

$$
(1 / 2)<h(V, V) / g(V, V)<2
$$

for all nontrivial tangent vectors $V$, is a $C^{0}$ open subset of $\operatorname{Riem}(M)$ and all such metrics $h$ are also complete (respectively, incomplete). Notice that this same argument fails for nonpositive definite metrics $g$ because of the existence of null vectors. In fact, examples 
due to Williams [23] show that both geodesic completeness and geodesic incompleteness may fail to be stable for Lorentzian manifolds. Furthermore, this failure of stability may hold for compact as well as noncompact manifolds. Among other things, Williams showed that the torus $M=S^{1} \times S^{1}=\{(x, y) \mid 0 \leq x \leq 2 \pi, 0 \leq y \leq 2 \pi\}$ with the usual identifications and the complete flat metric $g=d x d y$ has arbitrarily close metrics $g_{n}=$ $d x d y+(1 / n) \sin (x) d y^{2}$ which are geodesically incomplete. In particular, direct calculation shows that the circle in $M$ corresponding to $x=0$ is an incomplete null geodesic for each $g_{n}$. In this example, the manifold and the metrics are all real analytic. Clearly, the metrics $g_{n}$ converge to the limit metric $g$ in the $C^{r}$-fine topology for every $r$.

Recall that the Christoffel symbols $\Gamma_{j}{ }^{i}{ }_{k}$ depend on the components $g_{i j}$ of the metric tensor and on the first partials of these components. Thus, metrics which are close in the $C^{1}$-fine topology will have geodesic equations which are close and hence geodesic systems which are close. The above mentioned example of Williams shows that requiring metrics to have close geodesic systems by using the $C^{1}$-fine topology on $\operatorname{Lor}(M)$ is not sufficient to guarantee the stability of completeness. The next result [1] gives sufficient conditions for the $C^{1}$-stability of completeness for causal geodesics in terms of causal disprisonment and causal pseudoconvexity.

THEOREM 3.2. Let $(M, g)$ be timelike and null geodesically complete. If $(M, g)$ is both causally disprisoning and causally pseudoconvex, then there is a fine $C^{1}$ neighborhood $W(g, \delta)$ of $g$ in $\operatorname{Lor}(M)$ such that each $h \in W(g, \delta)$ is also both timelike and null geodesically complete.

Using Lemma 3.1 we obtain the following corollary which applies to a number of important examples such as Minkowski space-time, Einstein static space-time, and De Sitter space-time.

Corollary 3.3. Let $(M, g)$ be timelike and null geodesically complete. If $(M, g)$ is globally hyperbolic, then there is a fine $C^{1}$ neighborhood $W(g, \delta)$ of $g$ in $\operatorname{Lor}(M)$ such that each $h \in W(g, \delta)$ is also both timelike and null geodesically complete.

The singularity theorems [12] imply that, under certain physically realistic assumptions, there must exist at least one causal geodesic which is incomplete. However, one of the assumptions used in these theorems is the timelike convergence condition which is the assumption that the Ricci curvature is nonnegative on all timelike vectors (i.e., $\operatorname{Ric}(V, V) \geq 0$ for all timelike vectors $V$ and hence also for all null vectors). This condition fails to be $C^{r}$-fine stable for all $r$, since if there is a vector $V_{0}$ with $\operatorname{Ric}\left(V_{0}, V_{0}\right)=0$, then one may always perturb the metric $g$ to get a metric $h$ with negative Ricci curvature for $V_{0}$ such that $h$ is arbitrarily close to $g$ in any given $C^{r}$-fine topology. Consequently, since geodesic incompleteness is not a stable property in general, one would like to obtain a stability result which applies under some physically reasonable assumption. The next result [2] gives a sufficient condition for the $C^{1}$-fine stability of geodesic incompleteness in terms of the failure of partial imprisonment. Recall that a geodesic $\gamma(a, b)$ fails to be partially imprisoned as $t \rightarrow b^{-}$if for each compact set $K$ there is a parameter value $t_{1}$ such that $\gamma(t)$ fails to lie in $K$ for all $t_{1}<t<b$. In other words, given any fixed compact set $K$, the geodesic $\gamma$ eventually leaves $K$ and never returns. 
TheOREM 3.4. Let $(M, g)$ be a Lorentzian manifold and assume that $(M, g)$ has a geodesic $\gamma(a, b) \rightarrow M$ which is incomplete in the forward direction (i.e., $b<\infty)$. If the forward end (i.e., corresponding to $t \rightarrow b^{-}$) is not partially imprisoned in any compact set, then there is a $C^{1}$-fine neighborhood $W(g)$ of $g$ in Lor $(M)$ such that each metric in $W(g)$ has at least one incomplete geodesic. Furthermore, if $\gamma$ is nonspacelike, then $W(g)$ may be chosen such that each $h \in W(g)$ has an incomplete nonspacelike geodesic.

Since each end of each causal geodesic in a strongly causal space-time fails to be partially imprisoned [12], one obtains the following corollary.

Corollary 3.5. Let $(M, g)$ be a strongly causal space-time. If $(M, g)$ has a least one incomplete causal geodesic, then there is a $C^{1}$-fine neighborhood $W(g)$ of $g$ in Lor $(M)$ such that each metric in $W(g)$ has at least one incomplete causal geodesic.

The Hadamard-Cartan Theorem [15] is a fundamental result of Riemannian geometry in the large. This theorem guarantees that a complete simply connected Riemannian manifold with no conjugate points has an exponential map which, at each fixed point $p$, is a diffeomorphism from the tangent space $T_{p} M$ onto the manifold $M$. Among other things, this yields that the manifold $M$ is topologically $R^{n}$. A complete simply connected Riemannian manifold is called a Hadamard manifold. For positive definite manifolds, nonpositive sectional curvature implies the nonexistence of conjugate points. Using this fact and well known results on covering manifolds, it follows that a complete Riemannian manifold with nonpositive sectional curvature has a universal covering space which is a Hadamard manifold.

In order to get a Lorentzian version of the Hadamard-Cartan Theorem, we first extend our notions of disprisoning and pseudoconvexity from the collection of causal geodesics to all geodesics. The Lorentzian manifold $(M, g)$ is said to have a geodesic system which is disprisoning if each end of each geodesic fails to be imprisoned in any compact set. Similarly, the Lorentzian manifold $(M, g)$ is said to have a geodesic system which is pseudoconvex if for each compact set $K$ there is another compact set $H$ such that all geodesic segments with both endpoints in $K$ have their images entirely contained in $H$. Our next result is a Lorentzian Hadamard-Cartan theorem. A somewhat strengthened version may be found in [6].

THEOREM 3.6. Let $(M, g)$ be a complete $n$-dimensional Lorentzian manifold with a geodesic system which is both disprisoning and pseudoconvex. If $(M, g)$ has no conjugate points, then $M$ is topologically $R^{n}$ and at each point $p \in M$ the exponential map is a diffeomorphism from its domain in $T_{p} M$ onto $M$. Furthermore, $M$ is geodesically connected.

4. The space of geodesics. In this section we let $M$ have dimension $n$ and consider the collection of all geodesics of $(M, g)$ where each geodesic is identified as a point in a new space $G(M)$. Here geodesics are identified if they represent different parametrizations of the same point set. Thus, $\gamma_{1}$ and $\gamma_{2}$ are identified if there are constants $a$ and $b$ with $a \neq 0$ such that $\gamma_{1}(t)=\gamma_{2}(a t+b)$. Clearly, this is an equivalence relation on the collection of geodesics of $(M, g)$. Let $[\gamma]$ denote the equivalence class of the geodesic $\gamma$. If $v$ is a 
nontrivial tangent vector to $M$ at some point $p \in M$, let $\gamma_{v}$ be the geodesic which satisfies $\gamma_{v}{ }^{\prime}(0)=v$. Let $T^{\prime} M$ denote the collection of all nontrivial tangent vectors of $M$ and let $\pi: T^{\prime} M \rightarrow G(M)$ be the map $v \rightarrow\left[\gamma_{v}\right]$. We put a topology on the space of geodesics $G(M)$ by requiring that $\pi$ be a quotient map. Thus, a subset $W$ of $G(M)$ is open iff $\pi^{-1}(W)$ is open in $T^{\prime} M$.

An alternative approach to the topology on $G(M)$ is given by tangential convergence. A sequence of geodesics $\left\{\gamma_{n}\right\}$ is said to converge tangentially to the geodesic $\gamma:(a, b) \rightarrow M$ if there is some parameter value $a<t_{0}<b$ and a sequence of geodesics $\left\{\beta_{n}\right\}$ with $\beta_{n}$ $\in\left[\gamma_{n}\right]$ for each $n$ and with $\beta_{n}^{\prime}\left(t_{0}\right) \rightarrow \gamma^{\prime}\left(t_{0}\right)$. Notice that this is the requirement that there is some point $\gamma\left(t_{0}\right)$ such that corresponding points of the geodesics $\gamma_{n}$ converge to this point and the the tangents at the corresponding points of the geodesics $\gamma_{n}$ converge in direction to the direction determined by $\gamma^{\prime}\left(t_{0}\right)$. It can be shown that the sequence $\left\{\gamma_{n}\right\}$ converges tangentially to $\gamma$ iff the sequence $\left[\gamma_{n}\right]$ in $G(M)$ converges to $[\gamma]$. Thus, convergence in the quotient topology on $G(M)$ is equivalent to tangential convergence of geodesics of $(M, g)$. Using tangential convergence one may prove that $G(M)$ is a $T_{1}$ topological space iff the image of each geodesic of $(M, g)$ is a closed subset of $M$. It follows that the Einstein Static Universe and anti-DeSitter space-time have spaces of geodesics which are $T_{1}$. However, the spaces of geodesics for these space-times are not Hausdorff, compare [5].

We will say that $(M, g)$ has the nonreturning property if each point $p \in M$ has arbitrarily small neighborhoods such that any geodesic which leaves such a neighborhood fails to return. In other words, the nonreturning property is the natural extension of strong causality to the collection of all geodesics. The following result [5] shows that for manifolds which are nonreturning, pseudoconvexity is equivalent to each of $G(M)$ being Hausdorff and $G(M)$ being a $2 n-2$ dimensional manifold.

THEOREM 4.1. If $M$ is $n$-dimensional and $(M, g)$ is nonreturning, then the following three conditions are equivalent: (1) $G(M)$ is a Hausdorff topological space; (2) $G(M)$ is a $2 n-2$ dimensional manifold; and (3) the geodesic structure of $(M, g)$ is pseudoconvex.

If $\left(M_{1}, g_{1}\right)$ and $\left(M_{2}, g_{2}\right)$ are semi-Riemannian manifolds of arbitrary metric signature and arbitrary dimensions, the natural product metric on $M_{1} \times M_{2}$ is the semi-Riemannian metric given by $g=g_{1} \oplus g_{2}$. Recall that a topological space $X$ is said to be locally Euclidean if each $p \in X$ has a neighborhood $U(p)$ which is homeomorphic to an open set in some $R^{n}$. A locally Euclidean space need not be Hausdorff and thus need not be a manifold. Our next result [5] gives conditions for $G\left(M_{1} \times M_{2}\right)$ to be locally Euclidean.

THEOREM 4.2. Let $\left(M_{1}, g_{1}\right)$ and $\left(M_{2}, g_{2}\right)$ be semi-Riemannian manifolds and assume that both $G\left(M_{1}\right)$ and $G\left(M_{2}\right)$ are locally Euclidean. Then $G\left(M_{1} \times M_{2}\right)$ is locally Euclidean iff each of $\left(M_{1}, g_{1}\right)$ and $\left(M_{2}, g_{2}\right)$ fails to have any closed geodesics.

It can be proven [5], that if $\left(M_{1}, g_{1}\right)$ and $\left(M_{2}, g_{2}\right)$ are each nonreturning and pseudoconvex semi-Riemannian manifolds, then $G\left(M_{1} \times M_{2}\right)$ is a manifold. On the other hand, this result fails for warped products. In particular, universal two dimensional anti-DeSitter space-time is given by $d s^{2}=-\cosh ^{2}(x) d t^{2}+d x^{2}$ on $R^{2}$ and the geodesic space of this space-time fails to be Hausdorff and hence fails to be a manifold. Since this space-time 
is clearly a warped product of $\left(R^{1}, d x^{2}\right)$ with $\left(R^{1},-d t^{2}\right)$ using the warping function $\cosh ^{2}(x)$, we find that $G\left(M_{1} \times_{f} M_{2}\right)$ need not be a manifold even when $\left(M_{1}, g_{1}\right)$ and $\left(M_{2}, g_{2}\right)$ are nonreturning and pseudoconvex.

5. Geodesics and curvature. Any study of geometry in the large must involve both geodesics and curvature because of the fundamental roles they play in understanding global questions. We have compared and contrasted global results for positive definite metrics with the corresponding results for metrics which are indefinite. One underlying difficulty in the investigation of metrics which fail to be definite is that the sectional curvature function for these spaces becomes generically unbounded near degenerate sections, see [3], [4]. Since the sectional curvature is a key tool in studying the behavior of geodesics, this difficulty with the sectional curvature function greatly complicates the global study of manifolds which have metrics which are indefinite. This sectional curvature difficulty also has a physical implication for space-times. Observers moving close to the speed of light, generically, experience large tidal accelerations. Another fundamental obstacle in the investigation global questions in the indefinite case is that there is no fully satisfactory replacement for the Hopf-Rinow Theorem. In particular, the conclusions of the Hopf-Rinow Theorem fail to hold for complete Lorentzian manifolds. Thus, it is clear that one needs to have conditions such as global hyperbolicity for these spaces to use as at least partial replacement for the completeness assumption used in the Riemannian case. We have seen that for many applications one may use an alternative assumption of pseudoconvexity as a substitute for a completeness assumption. The study of the space of geodesics $G(M)$ illustrates the fundamental role that pseudoconvexity plays in determining the geodesic structure. Future investigations will involve investigating how the curvature determines the topological structure of $G(M)$ and how curvature is related to the pseudoconvexity property.

\section{References}

[1] J. K. Beem and P. E. Ehrlich, Geodesic completeness and stability, Math. Proc. Camb. Phil. Soc. 102 (1987), 319-328.

[2] J. K. Beem, P. E. Ehrlich, and K. L. Easley, Global Lorentzian Geometry, Second Edition, Pure and Applied Math. Vol. 202, Marcel Dekker, New York, 1996.

[3] J. K. Beem and S. G. Harris, The generic condition is generic, Gen. Rel. and Grav. 25 (1993), 939-962.

[4] J. K. Beem and S. G. Harris, Nongeneric null vectors, Gen. Rel. and Grav. 25 (1993), 963-973.

[5] J. K. Beem, R. J. Low, and P. E. Parker, Spaces of geodesics: products, coverings, connectedeness, Geometriae Dedicata 59 (1996), 51-64.

[6] J. K. Beem and P. E. Parker, Pseudoconvexity and geodesic connectedness, Annali Math. Pura. Appl. 155 (1989), 137-142.

[7] J. K. Beem and P. E. Parker, Sectional curvature and tidal accelerations, J. Math. Phys. 31 (1990), 819-827. 
[8] P. T. Chruściel, On Uniqueness in the Large of Solutions of Einstein Equations ("Strong Cosmic Censorship"), Australian University Press, Canberra, 1991.

[9] G. Galloway, The Lorentzian splitting theorem without completeness assumption, J. Diff. Geom. 29 (1989), 373-387.

[10] G. Galloway, The Lorentzian version of the Cheeger-Gromoll splitting theorem and its applications to General Relativity, in Differential Geometry: Geometry in Mathematical Physics and Related Topics, ed. R. Greene and S. -T. Yau, Amer. Math. Soc. Proceedings of Symposia in Pure Math., Vol. 54, Part 2 (1993), 249-257.

[11] D. Gromoll, W. Klingenberg and W. Meyer, Riemannsche Geometrie im Grossen, Lecture Notes in Math. Vol. 55, Springer-Verlag, Berlin, 1975.

[12] S. W. Hawking, G. F. R. Ellis, The Large Scale Structure of Space-time, Cambridge University Press, Cambridge, 1973

[13] N. J. Hicks, Notes on Differential Geometry, D. Van Nostrand, Princeton, New Jersey, 1965 .

[14] H. Hopf and W. Rinow, Über den Begriff des vollständigen differentialgeometrischen Fläche, Comment. Math. Helv. 3 (1931), 209-225.

[15] S. Kobayashi, Riemannian manifolds without conjugate points, Ann. Math. Pura. Appl. 53 (1961), 149-155.

[16] A. Królak, Black holes and weak cosmic censorship, Gen. Rel. and Grav. 16 (1984), 365-373.

[17] A. Królak and W. Rudnicki, Singularities, trapped sets, and cosmic censorship in asymptotically flat space-times, International J. Theoret. Phys. 32 (1993), 137-142.

[18] B. Mashhoon, Tidal radiation, Astrophys. J. 216 (1977), 591-609.

[19] B. Mashhoon and J. C. McClune, Relativistic tidal impulse, Month. Notices Royal Astron. Soc. 262 (1993), 881-888.

[20] R. P. A. C. Newman, A proof of the splitting conjecture of S.-T. Yau, J. Diff. Geom. 31 (1990), 163-184.

[21] B. O'Neill, Semi-Riemannian Geometry with Applications to Relativity, Pure and Applied Math. Vol. 103, Academic Press, New York, 1983.

[22] H. -J. Seifert, Global connectivity by timelike geodesics, Zs. f. Naturforsche 22a (1967), $1356-1360$.

[23] P. M. Williams, Completeness and its stability on manifolds with connection, Ph.D. Thesis, Dept. Math. Univ. of Lancaster, 1984. 